\title{
La relación entre la discapacidad intelectual, la inteligencia y la eugenesia en la América del siglo XX
}

\section{The relationship between intellectual disability, intelligence and eugenic in the America of the 20th Century}

\section{Palabras clave}

Discapacidad intelectual, inteligencia, eugenesia, conocimiento, retardo mental.

\section{Keywords}

Intellectual disability, intelligence, eugenic, knowledge, mental retardation.

\section{Melissa Stefania Cruz \\ Puerto \\ <mscruzp@unal.edu.co>}

Universidad Nacional de Colombia. Colombia

\section{Introducción}

El diagnostico de discapacidad intelectual tiene la particularidad de que no da cuenta de un problema individual, sino de la forma en que la normalidad se construye para crear el problema de la persona con discapacidad (Davis, 2006). Para dar cuenta de dicha construcción, este trabajo argumenta que la discapacidad toma un nuevo valor social y político a partir de los procesos sociales, discursivos y prácticos producto de la industrialización a finales del siglo XIX, y su posterior desarrollo de la mano con los discursos eugenésicos y las diversas teorías y técnicas sobre la medición de la inteligencia propios del siglo XX. Para Davis (2006), este cambio se basó en una falsa noción de cuerpo político-productivo en aras de la acumulación de capital para la burguesía; es decir, el cuerpo del trabajador se convirtió en parte del engranaje de los procesos de producción. Con base en lo cual, este trabajo busca presentar una reconstrucción histórica que reflexione la relación entre los conceptos de discapacidad intelectual, retardo mental, eugenesia y poder en la América del siglo XX, haciendo un énfasis en el desarrollo en Colombia.

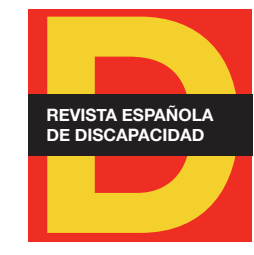

Para citar:

Cruz, M. (2020). "La relación entre la discapacidad intelectual, la inteligencia y la eugenesia en la América del siglo XX". Revista Española de Discapacidad, 8(I), pp. 261-271.

Doi: <https://doi.org/10.5569/23405104.08.01.15> 


\section{La relación de la inteligencia y la eugenesia}

La situación política y social en Europa, particularmente en Inglaterra, a mediados del siglo XIX fue propicia para la consolidación de la idea de jerarquización social y, por ende, de la existencia de individuos carentes y diferentes a partir de sus capacidades (Palma, 2005). Palma (2005) también señala que Inglaterra, al ser uno de los imperios coloniales más grande y tener bajo su control grandes territorios en Asia y África, contaba con un número significativo de proletariado explotado en minas, hilanderías y fábricas, sumido en la pobreza, la miseria y la promiscuidad. Esta situación contrastaba con los valores cristianos universales que profesaba el imperio, los cuales partían de la idea de que los hombres estaban hechos a imagen y semejanza del creador, generando una contradicción lógica entre los ideales cristianos de igualdad y caridad y las prácticas propias de la economía imperial victoriana. Esto produjo el marco preciso para la consolidación de las ideas biologicistas y sociológicas del darwinismo social y posterior modelo eugenésico, con base en las cuales las diferencias sociales eran entendidas y definidas como fenómenos naturales de adaptación biológica y social del más apto (Palma, 2005).

Dicho cambio permitió la apropiación política de los principios de la sociedad liberal y la consolidación de la idea de superioridad racial. A partir de allí, el éxito social y económico de una persona se convirtió en un parámetro para estimar su valor dentro de la sociedad, quedando implícita la idea de que "los ricos lo son porque son más listos, y los pobres lo son porque son menos eficientes o tontos” (Palma, 2005: 39); lo que implicó dejar de lado posturas morales, sociales y políticas que entraban a criticar el modelo de explotación colonial desde el ideal de igualdad de los seres humanos para justificar la distribución desigual de los recursos en términos biológicos.

El discurso de la eugenesia ha estado siempre relacionado con la expectativa de la sociedad idealizada, en la que sólo prevalecen las características socialmente valoradas por los grupos con poder. Dicho discurso se institucionaliza a partir de los aportes de Francis Galton, quien es considerado uno de los padres de la antropometría, la genética cuantitativa y la psicometría. Este autor definió la eugenesia como "la ciencia que trata todas las influencias que mejoran las cualidades innatas, o materia prima, de una raza, también aquellas que la pueden desarrollar hasta alcanzar la máxima superioridad" (citado por Ruiz y Suárez, 2000: 84). Con base en esta definición, su trabajo se convirtió en una búsqueda por lograr entender la herencia de las cualidades, carácter y habilidades de generación en generación, con el fin de maximizar la superioridad de la raza blanca occidental (Valencia, 2014). El trabajo de Galton concluye que el hecho de comprender dicha evolución de forma individual, sujeto por sujeto, era imposible y, por el contrario, la empresa científica y social se debería basar en medir las variaciones y sus efectos en grupos poblacionales utilizando como herramienta la estadística (Valencia, 2014).

La popularidad que alcanzó la teoría evolutiva de Darwin en el ámbito social generó que se popularizaran y confundieran los términos aptitud y mérito, quedando vinculadas características como la inteligencia con la laboriosidad y la decencia (Aiken, 2003). La inteligencia fue el rasgo principal en el que se centró Galton en su empresa eugenésica, siendo su objetivo determinar si rasgos mentales como la inteligencia se transmitían de padres a hijos, con el fin de confirmar la teoría de que mientras los hombres eminentes victorianos tenían hijos igualmente eminentes y sobresalientes intelectual y socialmente, los hombres del proletariado, delincuentes, idiotas o débiles mentales tenían hijos con sus mismas características individuales y sociales 
negativas (Davis, 2016). La investigación de Galton se caracterizó por el uso de método genealógicos, como la construcción de árboles familiares, las entrevistas para el rastreo familiar de capacidades y el diseño de varias pruebas de discriminación sensorial y de reacción a diferentes componentes (Aiken, 2003). Lo anterior, con el fin de consolidar la idea proveniente del darwinismo social de que las capacidades mentales tanto positivas como negativas eran hereditarias y no podrían ser modificadas por el ambiente, generando una base biológica que sustentará y acreditará toda una amalgama de prejuicios y estereotipos sociales y raciales.

Estos planteamientos fueron la base para el surgimiento de la psicología diferencial y de la medición de la inteligencia. Aunque Galton es el gestor de dicha empresa, son el psicólogo Alfret Binet y el psiquiatra Théodore Simon (ambos franceses), quienes en 1905 crearon el primer test de inteligencia como mecanismo estandarizado para su medición. El objetivo principal de Binet y Simon, el primero ejerciendo su rol como Comisionado de Educación francés y por encargo del Ministerio de Educación de su país, era detectar alumnos con problemas de desempeño escolar para ver sus posibilidades de educación y escolarización (Rodríguez, 2012). Estos autores construyeron un test de inteligencia agrupando un conjunto de problemas y actividades que eran capaces de resolver los niños en diferentes grados escolares, atribuyéndose a las personas una edad mental y la edad cronológica (Rodríguez, 2012). La primera, daba cuenta de la relación entre lo que lograba hacer una persona en una prueba concreta en comparación con la media poblacional,y la segunda planteaba la edad que tenía la persona.

Contrario a la consolidación en la época del discurso eugenésico y de la gran popularidad del darwinismo social, de acuerdo con Rodríguez (2012), la investigación de Binet y Simon sobre la inteligencia se caracterizó por ser un trabajo que, lejos de querer dar cuenta de características y mecanismos hereditarios, buscaba un mecanismo de equiparación de oportunidades para las personas consideradas en esa época como débiles mentales o idiotas. El nacimiento de los test de inteligencia viene de la mano de una concepción de la inteligencia como ciertas características moldeables por el entorno y ligadas a procesos de educación y escolarización. Binet planteósu miedo a que este mecanismo de medición de la inteligencia fuera utilizado para fines de segregación y etiqueta de los niños en las aulas de clase por parte de partidarios de la eugenesia (Palma, 2005). En palabras de Binet: "He aquí una excelente ocasión para deshacernos de todos los niños que nos causan problemas, y sin auténtico sentido crítico englobar a todos los que son rebeldes o no demuestran interés por la escuela" (1905, citado por Gould, 1980:151).

A partir de los tres principios de Binet, Gould (1980) plantea una crítica a la teorías de inteligencia afirmando que éstasse basan en dos falacias: la primera, la falacia de cosificación, la cual implica que la inteligencia es una entidad determinada con unas características particulares, distinguibles y medibles a través de escalas discretas en las que cada sujeto se podría ubicar; la segunda, la falacia de la heredabilidad, que plantea que la inteligencia es una característica heredable y situada en los genes, que pasa de generación en generación como un rasgo dominante. Estas dos falacias han sido el sustento para la consolidación de la medición de la inteligencia como un argumento eugenista y política para fundamentar la supremacía de la raza blanca, estrato medio-alto norteamericana (Gould, 1980).

El concepto de $\mathrm{Cl}$ (cociente intelectual) se convirtió en uno de los pilares para la consolidación de la eugenesia en el discurso político y social occidental. El norteamericano Henry H. Goddard afirmó en una conferencia en la Universidad de Princeton en 1919 que los resultados de Cl bajos en la prueba de Binet-Simon 
eran la razón de la desigualdad social y del por qué unas personas eran ricas y otras pobres, por lo cual no era posible pensar en la posibilidad de una sociedad igualitaria, ya que las riquezas estaban distribuidas de acuerdo al $\mathrm{Cl}$ de las personas, es decir, de acuerdo a una entidad biológica, innata e inmutable como se entendía en esa época la inteligencia (Palma, 2005).

El trabajo de Goddard, de acuerdo con Gould (1980), sobre la inteligencia se dio en el tiempo en que la comunidad académica estaba altamente entusiasmada por el redescubrimiento de la obra de Mendel sobre las bases de la herencia. Este momento se caracterizó por la creencia generalizada de que todos los rasgos del ser humano, tanto orgánicos y físicos como comportamentales, se derivaban de un único gen. Esta postura fue rápidamente tomada por los eugenistas de la época, adoptando la idea de que los rasgos indeseables de las sociedades que se buscaban eliminar se debían a un único gen que podría controlarse a través de la restricción de la reproducción. Entre los rasgos deseables determinados por los eugenistas, la inteligencia tuvo una relevancia dentro del discurso social norteamericano y en parte europeo, en tanto fue considerado como un rasgo dominante que se podría transmitir de padres a hijos de manera directa (Gould, 1980).

Para ejemplificar mejor la importancia de la inteligencia en pleno auge de la industrialización, Goddard presento el estudio genealógico del caso de Martín Kallikak (citado por Carlson, 2006) analizaba su descendencia producto de la unión con su esposa, mujer burguesa, y de la relación extramatrimonial con una campesina. Las conclusiones de dicho estudio se pueden simplificar en el hecho de que los descendientes de la unión con su esposa fueron personas denominadas prestantes según los estándares de la época (abogados, buenos padres, médicos), mientras la descendencia de la unión con la mujer campesina produjo personas que no cumplían dichos estándares (ladrones, estafadores, cretinos). A partir de estos datos, se afirmó que los problemas de debilidad mental y perversidad eran hereditarios y se debían al "plasma germinal defectuoso o malasangre" (Carlson, 2006: 73) que pasaban de generación en generación; si un padre era débil mental todos sus hijos nacerían con la misma condición.

Adicionalmente, se sustentó que dichas capacidades mentales y morales inferiores estaban relacionadas directamente con el estatus social y económico de las personas, siendo estas características predominantes entre la clase campesina, obrera y los extranjeros (Davis, 2016). Debido a que la causa y el resultado de esta forma de discapacidad eran invisibles (la sangre y la baja inteligencia) se comenzaron a gestar proyectos y soluciones desde las instituciones de salud pública (la esterilización, la restricción del matrimonio, leyes de inmigración y la institucionalización), con el fin de evitar la propagación de dichos rasgos mentales deficientes (Carlson, 2006), a través de la consolidación de la eugenesia como un discurso y práctica social ampliamente acogido entre la clase burguesa.

En este contexto, el test de inteligencia de Binet-Simon se comenzó a usar de forma masiva en colegios para la medición de estudiantes y en comunidades de inmigrantes, personas afrodescendientes, nativos y entre otras comunidades minorizadas, con el fin de justificar su posición social inferior a partir de la premisa de la supervivencia del más apto (Gould, 1980). En 1912, William Stern introduce en el test de Binet-Simon el concepto decociente intelectual $(\mathrm{Cl})$, el cual es el resultado de dividir la edad mental por la edad real y multiplicar el resultado por 100, proporcionando una medida única de la inteligencia (EM/EC x 100) (Chávez, 2007: 4) y un parámetro de la media de la inteligencia del ser humano, postulado como un Cl igual a 100. La fascinación y amplio uso que se le dio al test de Binet-Simon en Norteamérica fue de la mano del psicó- 
logo Lewis Terman, quien lo modificó para que no fuera solo para niños y jóvenes sino también para adultos, equiparando edad cronológica y edad mental a 100 e incluyendo una desviación normal de 15; a este cambio se le denominó test de Stanford-Binet, ya que en esa época Terman era profesor de la Universidad de Standford (Rodríguez, 2012). Igualmente, Terman aplicó de forma masiva su modificación del test original de Binet-Simon en Norteamérica y con base en sus resultados aseguró que "los hispanoindios y los negros contaban con bajos niveles de inteligencia y predijo que en el futuro se hallarían aún más diferencias raciales enormemente significativas que no podrían ser suprimidas mediante ningún esquema de cultura mental" (Rodríguez, 2012: 13).

Para describir el panorama de las principales aproximaciones al concepto de inteligencia a partir de la aplicación de pruebas estandarizadas en el siglo XX, de acuerdo con Reschly et al. (2002), es posible dividirlas en dos grandes grupos: quienes creen que se caracteriza por un solo factor general (Spearman y Cattell) y quienes creen que son un conjunto de habilidades mentales (Thurstone y Gardner). Adicionalmente, existe un tercer cuerpo de teorías que plantean la inteligencia de la mano con el desarrollo de estructuras intelectuales (Piaget y Vygotsky) y habilidades mentales. Estas aproximaciones se caracterizan por afirmar que las pruebas de inteligencia son las mejores formas de predecir repertorios conductuales y habilidades de las personas, tanto en la parte escolar como en su vida diaria (Reschlyet al., 2002). Desde este panorama se presentan las principales aproximaciones al concepto de inteligencia a partir de la aplicación de pruebas estandarizadas.

\section{El contexto de Latinoamérica y Colombia}

Desde este marco, Latinoamérica y Colombia no fueron la excepción frente a los discursos de la eugenesia a finales del siglo XIX y principios del siglo XX. Según McGraw (2007), las naciones latinoamericanas, recién independizadas de la colonización española, quedaron a merced de nuevas clases de colonialidad e imperialismo que generalmente venían en forma de superioridad racial. Este nuevo ambiente independentista y de apertura social y económica, generó una nueva clase intelectual que proclamaba la distinción entre los latinos y los anglosajones y la superioridad racial (McGraw, 2007). Ese escenario fue propicio para los discursos eugenésicos de la mano de agentes como la iglesia católica y las elites económicas, ya que permitía la posibilidad de una nueva forma de colonialidad a través de la búsqueda de unidad racial a partir de justificaciones científicas y biológicas (McGraw, 2007).

En Colombia particularmente, de acuerdo con Castro-Gómez y Grosfoguel (2007), la colonialidad del poder se instaura con el deseo de los colonos de parecerse al conquistador europeo, en busca de verse y definirse a partir de la imagen de este en el siglo XVII. Los criollos, indios, mestizos y negros eran denominados como de "sangre de la tierra" (Castro-Gómez, 2007: 85), y los españoles y descendientes europeos eran considerados como blancos, no tanto por su color de piel sino por la limpieza de su sangre. La pertenencia a la casta de blancos era demostrada públicamente a través del tipo de vestuarios, el matrimonio católico, el uso del don, el tipo de oficio que desempeñaba, el lugar de vivienda y la ostentación de un título universitario (Castro-Gómez y Grosfoguel, 2007). Por ejemplo, uno de los requisitos para el ingreso a la Universidad era 
tener "sangre limpia", ascendencia española y no tener ninguna relación con la "sangre de la tierra", como eran denominadas a las comunidades indígenas, tales condiciones se convirtieron en un requisito circular que operaba como mecanismo de legitimación del capital cultural heredado por las elites, mediante la segregación etnoracial (Castro-Gómez y Grosfoguel, 2007).

A diferencia de Europa y Norteamérica, en Colombia y Latinoamérica el discurso eugenésico no estuvo ligado con el concepto de inteligencia sino con el concepto de raza, debido al pasado colonial y a la diversidad cultural y étnica que caracteriza la región. La eugenesia se planteó como un problema de superioridad racial en términos de color de piel y características culturales propias de las regiones, siendo una condición para obtener una posición social ventajosa el seguir el espejo del conquistador en tanto blanqueamiento de la raza (Maldonado-Torres, 2007). Por ejemplo, diferentes investigaciones y discursos sociales en Colombia a finales del siglo XIX y principios del siglo XX, conllevaron a consolidar la representación de la población de las costas colombianas (los costeños) como "un grupo en condiciones morales y culturales no aptas para la ciudadanía" (McGraw, 2007: 63), no solo por las condiciones ambientales y geográficas de la región sino por las características etnoraciales de sus pobladores.

A finales del siglo XIX e inicios del siglo XX, al mismo tiempo que la eugenesia toma un gran auge en Latinoamérica, el establecimiento de la tutela y el patronato estatal se consolidan como una salida política y social al problema de la población infantil desescolarizada y vulnerable, como lo eran los niños y niñas pobres, huérfanos, abandonados, delincuentes, viciosos, débiles mentales e idiotas, lo que conllevó a que estos niños y jóvenes fueran institucionalizados en diferentes lugares de acogida (Valencia, 2004). Esto como una medida de control higienista para la protección de la raza en términos de asegurar las condiciones ambientales a las que estos niños están expuestos.

En el marco de América Latina, Argentina se convierte en un caso particular en el cual las ideas eugenistas se combinaron por la preocupación por la educabilidad y rehabilitación de niños y niñas débiles mentales o con otro tipo de patologías, a partir de los principios lamarckianos de la heredabilidad de los caracteres adquiridos (Tovar, 2016). Según Valencia (2014), las políticas eugenistas en este país se caracterizaron por su connotación positiva, por la idea de que la relación con el entorno puede transformar características biológicas y eliminarlas, previniendo su heredabilidad y justificando la consolidación de la educación y escuelas especiales para niños y niñas débiles mentales. Estas escuelas posteriormente son reemplazadas por colonias de verano, ya que se consideraba que los climas húmedos favorecen prácticas de fortalecimiento de sus debilidades debido al aire que se respiraba (Valencia, 2014).

Tovar (2016) afirma que los debates eugenésicos en Colombia tuvieron sus inicios motivados por los acuerdos realizados en La Habana en 1927 y se caracterizaron por discursos que buscaban implementar medidas como el certificado médico prenupcial; acciones de higiene social; programas de higiene y salud para mujeres embarazadas, niños y niñas recién nacidos, y niños y niñas escolarizados; implementación de la educación física como medida de fortalecimiento físico de la raza; entre otras formas de control. Dichos discursos se basaron en una visión neo-lamarckiana, la cual afirmaba que los rasgos adquiridos son heredables, lo que generó que desde las elites políticas e intelectuales se buscarán los factores externos que llevaban a la degeneración de la raza, para posteriormente postular soluciones sociales y políticas para la renovación racial del país. 
En 1916 el médico Miguel Jiménez López, en la conferencia inaugural de la cátedra de clínica de patología mental en la Facultad de Medicina de la Universidad Nacional de Colombia, planteó que las enfermedades mentales eran producto de la herencia racial en Colombia, debido al cruce entre una raza violenta como la europea con la raza chibcha, considerada como degenerada por el consumo del alcohol; este cruce sólo podría producir una raza deficiente e impotente (citado por Beltrán, 2015: 20).

En este contexto latinoamericano la deficiencia se puede entender como una retórica social, histórica y cultural relacionada con la ética de la normalidad "que regulan y controlan la forma acerca de cómo son pensados los cuerpos y las mentes de los otros" (Skliar, 2010: 113). Skliar (2010) adicionalmente retoma una cita de McLaren, en lo que se evidencia cómo la deficiencia, entendida desde el discurso de la educación especial, lleva a procesos sociales y políticos diferentes de acuerdo con la posición social de las personas. El funcionamiento diferencial de políticas de significación de la deficiencia lleva a que en América Latina una gran proporción de estudiantes negros y latinos de clase baja se etiqueten como con "problemas de conducta", mientras que la mayor parte de estudiantes blancos clase media se les etiquete como con "problemas de aprendizaje" (citado por Skliar, 2010: 114).

Skliar (2000) resalta este aspecto, al señalar la similitud entre los discursos colonialistas europeos que se refieren a la naturaleza de los africanos o de las tribus indígenas o de los hispanos y textos que tratan sobre personas con discapacidad intelectual y auditiva, "en los cuales se nombran, por igual, la ausencia de lenguaje, la inteligencia primitiva, la inmadurez afectiva y cognitiva, y los comportamientos agresivos y peligrosos" (Skliar: 114), que conllevan conjuntamente procesos de medicalización y paternalismo.

\section{Consolidación del diagnóstico de discapacidad intelectual}

Los cambios de la discapacidad intelectual como diagnóstico y concepto han pasado de visiones religiosas en donde la diversidad cognitiva era producto del pecado o del demonio, a posturas biologicistas iniciales, como las planteadas en el anterior apartado, relacionadas con discursos eugenésicos y hereditarios, hasta las concepciones netamente psicométricas de principios del siglo pasado, las cuales establecieron límites numéricos y medibles para determinarlo. Estos cambios, a partir de los años 70, se consolidaron en una búsqueda por caracterizar el llamado retraso mental como una unidad diagnóstica que no solo diera cuenta de parámetros psicométricos sino también de la dimensión relacional de los individuos de la mano con el naciente modelo social de la discapacidad.

Contrario al desarrollo del concepto de inteligencia, Verdugo (1994) plantea cómo el desarrollo del concepto de retardo mental estuvo relacionado con una visión más social y relacional de la interacción del individuo con su entorno, resaltando la concepción clásica de retardo postulada por Tredgold (1937), quien lo concibe como la incapacidad de llevar una vida adulta independiente, y la divide en tres grupos: alteración mental, deterioro mental y desarrollo incompleto (citado por Verdugo 1994). Verdugo (1994), señala que otros autores como Doll $(1941,1953)$ y Kanner (1957) sugirieron que esta definición debería estar acompañada por la falta de habilidades sociales, madurez y dependencia del sujeto (citado por Verdugo 1994). 
A partir de esta nueva concepción del diagnóstico desde un modelo social y relacional, la American Association on Intellectual and Developmental Disabilities (AAIDD, 2002) comienza a plantear cambios significativos relevantesen la concepción sobre el retraso mental entre los cuales se encuentran:

- El retraso mental implica un estado de funcionamiento.

- Reformulaba y clasificaba los sistemas de apoyo que se debían presentar y su intensidad.

- Entendía el retraso mental como el resultado de la interacción del individuo con un funcionamiento limitado y el entorno, dejando de lado la perspectiva que entendía esto como un problema personal.

- Definía la conducta adaptativa como una serie de habilidades (conceptuales, sociales y prácticas) necesarias para la adaptación al entorno.

Sin embargo, en el marco de la reivindicación de los derechos de las personas con discapacidad y en el establecimiento de un modelo social de la discapacidad, el concepto de retraso mental evoluciona y se reemplaza por discapacidad intelectual, respondiendo a una construcción alrededor de la capacidad de adaptación de un individuo a su entorno (MEN, 2006). Dentro de este se ubica el concepto de discapacidad cognitiva como una disposición funcional de una serie de habilidades de procesamiento de información y estilos de aprendizaje (MEN, 2006). Sin embargo, esta organización conceptual no está completamente clara dentro de la bibliografía disponible, ni en los discursos cotidianos apropiados socialmente, lo que conlleva a que generalmente se igualen los conceptos, como si todos significaran e implicaran lo mismo, o a que se usen de forma indiscriminada sin tener en cuenta sus significados o particularidades.

Retomando la conceptualización de la AAIDD (2002), la discapacidad intelectual no remite a una observación y medición de rasgos clínicos observables sino a un concepto dinámico y funcional que responde al nivel de adaptación de las personas con las demandas de su entorno, si esta logra adaptarse a las circunstancias de sus contextos. A partir de esta caracterización, el diagnóstico y su evaluación remiten aspectos ecológicos y sociales de adaptación e integración con los diferentes sistemas relacionales, familia, colegio, comunidad, etc.

Este diagnóstico no recoge una patología particular sino por el contrario una expectativa de funcionamiento, tanto intelectual como social, dentro de las exigencias de la sociedad y la cultura. Este punto queda ejemplificado en el Manual de Clasificación de la CIE-10 de los Trastornos Mentales y del Comportamiento en Niños y Adolescentes (OMS, 2010), en donde la discapacidad intelectual está ubicada en el eje III, nivel intelectual, independiente de otras clasificaciones, debido a que no corresponde a ningún otro marco de clasificación nosotáxico, sino al concepto de inteligencia, el cual no es entendido como una función única sino como un número de capacidades específicas y equiparable entre individuos; si existe un grado de discrepancia de dichas capacidades en un individuo con relación a su entorno, se afirma que existe un retraso mental (González et al., 2006). 


\section{Conclusiones}

La construcción del concepto de la discapacidad ha variado históricamente de acuerdo los discursos dominantes, en el siglo XX el discurso del capital e industrialización es acogido dentro de los países en América, ya que la discapacidad se comienza a entender en términos de anormalidad y falta, frente al ideal del hombre occidental productor y consumidor del naciente capitalismo. La discapacidad intelectual como unidad diagnostica se caracteriza por un funcionamiento intelectual inferior a la norma y una deficiencia en las habilidades adaptativas de los sujetos frente a las expectativas sociales, lo que responde a los intereses que parametrizan la normalidad de acuerdo con los imaginarios y estereotipos que se quieren instaurar hegemónicamente. La normalidad, que representa al individuo neurotípico, que enaltece los discursos occidentales y modernos, es aquella que responde a las demandas del mercado y que caracteriza a un cuerpo productivo. En este sentido, la normalidad intelectual y cognitiva da cuenta de las habilidades de razonamiento, procesamiento de información y comunicación que se esperan dentro de las lógicas políticas y de mercado, y no una alteración fisiológica producida por procesos biológicos.

En este punto, la idealización neurotípica debería entender la inteligencia como una falta de adaptación a los contextos relacionales y sociales, comenzando por plantear discursos que aborden la desigualdad social, la exclusión y la discriminación a toda persona considerada como diferentes, abriendo la posibilidad para resistencias, voces subalternas y luchas comunes dentro del campo de los estudios críticos en discapacidad. Por lo que es necesario un reconocimiento de la discapacidad desde una postura crítica y social, que indague y cuestione las visiones simplistas desde marcos biomédicos, y permita abordajes relacionales y sociopolíticos de las realidades de las personas inmersas en este fenómeno. En este sentido, la discapacidad no debe ser el objeto último de la investigación, sino un dispositivo que recoge y estructura una serie de prácticas y discursos alrededor de relaciones de poder. Por ende, cualquier ruta teórica e investigativa que se establezca debe plantearse desde la transversalidad y la complejidad que permita entender la imbricación de factores sociales, históricos, políticos e individuales que configuran dicho dispositivo a través de instituciones como la escuela, comunidad, familia y sistema de salud. 
Referencias bibliográficas

Aiken, L. (2003). Tests psicológicos y evaluación. $11^{\text {a }}$ ed. México: Pearson.

American Association on Intellectual and Developmental Disabilities. (2002): Mental Retardation: Definition, Classification and Systems of Supports. 10th Ed. Washington DC: AAIDD.

Beltrán, J. (2015). Reflexiones sobre algunos alcances del racismo científico en Colombia. En W. Penagos (comp.), Educación en ciencias: experiencias investigativas en el contexto de la didáctica, la historia, la filosofía y la cultura. Bogotá: Universidad Distrital.

Carlson, L. (2006). The faces of intellectual disability. Indiana: Indiana University Press.

Castro-Gómez, S. y Grosfoguel, R. (2007). El giro decolonial. Bogotá: Siglo del Hombre.

Chávez, A. (2007). La Escala Stanford - Binet. Recuperado de https://comenio.files.wordpress.com/2007/09/binet_imprimir.pdf.

Davis, L. (2006). "Constructing Normalcy The Bell Curve, the Novel, and the Invention of the Disabled Body in the Nineteenth Century”. En L. Davis (ed.), The Disability Studies Reader. New York: Routledge.

González, D. et al. (2006). Nosotaxia y Nosología del Retraso Mental. VERTEX Revista Argentina de Psiquiatría, 17(2), pp. 373-377. Recuperado de http://www.itineris.org.ar/files/Nosolog\%C3\%ADa\%20y\%20Nosotaxia\%20del\%20Retraso\%20Mental.pdf.

Gould, S. J. (1980). The Mismeasure of man. Edición aumentada y revisada. Nueva York: W.W. Norton Company.

Maldonado-Torres, N. (2007). Sobre la colonialidad del ser: contribuciones al desarrollo de un concepto. En S. Castro-Gómez y R. Grosfoguel (2007), El giro decolonial: Reflexiones para una diversidad epistémica más allá del capitalismo global. Bogotá: Siglo del Hombre Editores.

McGraw, J. (2007). "Purificar la nación: eugenesia, higiene y renovación moral-racial de la periferia del Caribe colombiano, 1900-1930". Revista de Estudios Sociales, 27: 62-75.

Ministerio de Educación Nacional, MEN (2006). Orientaciones pedagógicas para la atención educativa a estudiantes con discapacidad cognitiva. Bogotá: Colombia Aprender. Recuperado de https://www.mineducacion.gov. co/1759/articles-360293_foto_portada.pdf.

Organización Mundial de la salud, OMS (2010). Clasificación multiaxial de los trastornos psiquiátricos en niños y adolescentes; clasificación de la CIE-10 de los trastornos mentales y del comportamiento en niños y adolescentes. Madrid: Editorial Médica Panamericana, S.A.

Palma, H. (2005). Gobernar es seleccionar. Historia y reflexiones sobre el mejoramiento genético en seres humanos. Buenos Aires: Jorge Baudino.

Reschly, D. et al. (2002). Mental Retardation: Determining eligibility for social security benefits. Washington, D.C.: National Academy Press.

Rodríguez, M. (2012). "Orígenes de los test de CI Una 'inteligencia a medida'”. Futuro, 22(157), pp. 12-18.

Ruiz, R. y Suárez, L. (2000). “Eugenesia y medicina social en el México posrevolucionario”. Revista Ciencias, 60(1), pp. 79-86. 
Skliar, C. (2000). "Discursos y prácticas sobre la deficiencia y la normalidad. Las exclusiones del lenguaje, del cuerpo y de la mente”. En P. Gentili (coord.), Códigos para la ciudadanía. La formación ética como práctica de la libertad. Buenos Aires: Santillana.

Tovar, J. (2006). La eugenesia en Colombia: aproximación bioética a un problema de justicia social (1900-1950). Bogotá: Universidad del Bosque.

Valencia, L. (2014). Eugenesia y test mentales: su aplicación en Estados Unidos y Argentina en la primera mitad del siglo XX. Recuperado de http://www.rebelion.org/docs/189172.pdf.

Verdugo, M. A. (1994). "El cambio de paradigma en la concepción del retraso mental: la nueva definición de la AAMR”. Siglo Cero, 25(5), pp. 5-24. 